\title{
ОЦЕНИВАНИЕ МАТРИЧНЫХ ПАРАМЕТРОВ КУСОЧНО-НЕПРЕРЫВНОЙ МОДЕЛИ НЕЛИНЕЙНОГО ОБЪЕКТА УПРАВЛЕНИЯ
}

\author{
Р.Л. Лейбов \\ Национальный исследовательский Московский государственный строительный университет, \\ г. Москва, РОССИЯ
}

Московский авиационный институт (национальный исследовательский университет), г. Москва, РОССИЯ

\begin{abstract}
Аннотация: В статье рассмотрены особенности оценивания матричных параметров кусочнонепрерывной модели по переходным процессам нелинейной модели объекта управления методом случайного поиска. Определен один из диапазонов возможного использования кусочно-непрерывной модели нелинейного объекта. Приведены результаты применения предлагаемого подхода при создании кусочно-непрерывной модели авиационного газотурбинного двигателя.
\end{abstract}

Ключевые слова: нелинейный объект, кусочно-непрерывная модель, переходные процессы нелинейной модели, метод случайного поиска, диапазон возможного использования кусочно-непрерывной модели

\section{NONLINEAR PLANT PIECEWISE-CONTINUOUS MODEL MATRIX PARAMETERS ESTIMATION}

\author{
Roman L. Leibov \\ National Research Moscow State University of Civil Engineering, Moscow, RUSSIA \\ Moscow Aviation Institute (National Research University), Moscow, RUSSIA
}

\begin{abstract}
This paper presents a nonlinear plant piecewise-continuous model matrix parameters estimation technique using nonlinear model time responses and random search method. One of piecewise-continuous model application areas is defined. The results of proposed approach application for aircraft turbofan engine piecewisecontinuous model formation are presented.
\end{abstract}

Keywords: Nonlinear plant, piecewise-continuous model, nonlinear model time responses, random search method, piecewise-continuous model application area

\section{1. ВВЕДЕНИЕ}

Для разработки алгоритмов управления, алгоритмов обнаружения, локализации, конкретизации и парирования отказов систем автоматического управления (САУ), а также для оценивания робастности этих систем и их алгоритмов используются линейные модели нелинейных объектов управления. Матричные параметры линейных моделей оцениваются по переходным процессам нелинейных моделей объектов управления. Несколько линейных моделей, матричные параметры которых должны оцениваться и сменяться друг другом согласованно, составляют кусочнолинейную модель нелинейного объекта. Кусочно-непрерывная модель позволяет построить кусочно-линейную модель, состоящую из произвольного числа согласованных друг с другом линейных моделей. Точность такой кусочнолинейной модели может быть максимально приближена к точности кусочнонепрерывной модели. В данной работе рассматриваются структура, особенности оценивания матричных параметров и один из диапазонов возможного использования кусочно-непрерывной модели нелинейного объекта управления. 


\section{2. НЕЛИНЕЙНАЯ, ЛИНЕЙНАЯ И КУСОЧНО-НЕПРЕРЫВНАЯ МОДЕЛИ НЕЛИНЕЙНОГО ОБЪЕКТА УПРАВЛЕНИЯ}

Нелинейная модель объекта управления, имеет вид [1], [2], [3]

$$
\dot{\mathbf{x}}=\mathbf{f}\left(\mathbf{x}^{\mathrm{p}}, \mathbf{u}^{\mathrm{p}}, \mathbf{w}^{\mathrm{p}}\right) .
$$

Здесь $\mathbf{x}^{\mathrm{p}}-$ вектор состояния, $\mathbf{u}^{\mathrm{p}}-$ вектор управления, $\mathbf{w}^{\mathrm{p}}-$ вектор внешних воздействий, a $\mathbf{f}-$ нелинейная вещественная векторная функция. Координаты вектора состояния (переменные состояния) такого объекта управления, например авиационного газотурбинного двигателя (ГТД), это частоты вращения роторов, температуры и давления в различных сечениях проточной части двигателя. Координаты вектора управления (переменные управления) - это расход топлива, изменяемые геометрические характеристики проточной части двигателя, в частности, площадь критического сечения реактивного сопла и углы поворота направляющих аппаратов вентилятора и компрессора. Координаты вектора внешних воздействий (переменные внешних воздействий) это угол отклонения рычага управления двигателем (управляющее воздействие), а также высота и скорость полета (переменные внешних условий). Постоянные управляющее воздействие и внешние условия, входящие в вектор $s \mathbf{w}$, определяют установившийся режим работы двигателя (рабочую точку), которому соответствуют вектор установившихся значений переменных управления su и вектор установившихся значений переменных состояния s $\mathbf{x}$.

В небольшой окрестности произвольного $\mathrm{r}$ ого установившегося режима нелинейный объект управления можно [1], [2], [3] приближенно описать с помощью линейной модели в нормированных отклонениях от установившихся значений

$$
\dot{\mathbf{x}}^{\mathrm{n}}={ }^{r} \mathbf{A}\left(\mathbf{x}^{\mathrm{n}}-{ }_{\mathrm{s}}^{r} \mathbf{x}^{\mathrm{n}}\right)+{ }^{r} \mathbf{B}\left(\mathbf{u}^{\mathrm{n}}-{ }_{\mathrm{s}}^{r} \mathbf{u}^{\mathrm{n}}\right),
$$

где $\mathbf{x}^{\mathrm{n}}-n$-мерный вектор нормированных переменных состояния, а $\mathbf{u}^{\mathrm{n}}-m$-мерный вектор нормированных переменных управления

$$
\begin{gathered}
x_{i}^{\mathrm{n}}=\frac{x_{i}}{\max \left|{ }_{\mathrm{s}} x_{i}\right|}, \quad i=1, \ldots, n, \\
u_{i}^{\mathrm{n}}=\frac{u_{i}}{\left.\max \right|_{\mathrm{s}} u_{i} \mid}, \quad i=1, \ldots, m,
\end{gathered}
$$

${ }^{r} \mathbf{A},{ }^{\prime} \mathbf{B}$ - матрицы динамической линейной модели для $r$-ого установившегося режима; ${ }^{r} \mathbf{A}, ' \mathbf{B}, r=1,2, \ldots, R$ матрицы динамической кусочно-линейной модели; ${ }_{\mathrm{s}}^{r} \mathbf{u}^{\mathrm{n}},{ }_{\mathrm{s}}^{r} \mathbf{x}^{\mathrm{n}}$, $r=1,2, \ldots, R \quad$ вектора статической характеристики объекта управления.

Пусть у кусочно-непрерывной модели вектора нормированных установившихся значений переменных управления ${ }_{\mathbf{s}} \mathbf{u}^{\mathrm{n}}$ и переменных состояния $\mathbf{s}^{\mathrm{n}}$, а также матрицы $\mathbf{A}$ и $\mathbf{B}$ определяются следующим образом

$$
\begin{array}{r}
{ }_{\mathrm{s}} \mathbf{u}^{\mathrm{n}}={ }_{\mathrm{s}}^{r} \mathbf{u}^{\mathrm{n}}+\theta\left({ }_{\mathrm{s}}^{r+1} \mathbf{u}^{\mathrm{n}}-{ }_{\mathrm{s}}^{r} \mathbf{u}^{\mathrm{n}}\right), r=1, \ldots, R-1, \\
{ }_{\mathrm{s}} \mathbf{x}^{\mathrm{n}}={ }_{\mathrm{s}}^{r} \mathbf{x}^{\mathrm{n}}+\theta\left({ }_{\mathrm{s}}^{r+1} \mathbf{x}^{\mathrm{n}}-{ }_{\mathrm{s}}^{r} \mathbf{x}^{\mathrm{n}}\right), r=1, \ldots, R-1, \\
\mathbf{A}={ }^{r} \mathbf{A}+\theta\left({ }^{r+1} \mathbf{A}-{ }^{r} \mathbf{A}\right), r=1, \ldots, R-1, \\
\mathbf{B}={ }^{r} \mathbf{B}+\theta\left({ }^{r+1} \mathbf{B}-{ }^{r} \mathbf{B}\right), r=1, \ldots, R-1,
\end{array}
$$

где $0 \leq \theta<1$, поскольку

$$
\begin{gathered}
\theta=\frac{\left\|\mathbf{x}^{\mathrm{n}}\right\|-\left\|{ }_{\mathrm{s}}^{r} \mathbf{x}^{\mathrm{n}}\right\|}{\left\|{ }_{\mathrm{s}}^{r+1} \mathbf{x}^{\mathrm{n}}\right\|-\left\|{ }_{\mathrm{s}}^{r} \mathbf{x}^{\mathrm{n}}\right\|}, \\
\frac{\left\|\mathbf{x}^{\mathrm{n}}\right\|}{\left\|{ }_{\mathrm{s}}^{R} \mathbf{x}^{\mathrm{n}}\right\|} \leq \frac{\left\|{ }_{\mathrm{s}} \mathbf{x}^{\mathrm{n}}\right\|}{\left\|{ }_{\mathrm{s}}^{R} \mathbf{x}^{\mathrm{n}}\right\|}, \quad r=1, \\
\quad \frac{\left\|{ }_{\mathrm{s}}^{r} \mathbf{x}^{\mathrm{n}}\right\|}{\left\|{ }_{\mathrm{s}}^{R} \mathbf{x}^{\mathrm{n}}\right\|} \leq \frac{\left\|\mathbf{x}^{\mathrm{n}}\right\|}{\left\|{ }_{\mathrm{s}}^{R} \mathbf{x}^{\mathrm{n}}\right\|} \leq \frac{\left\|{ }_{\mathrm{s}}^{r+1} \mathbf{x}^{\mathrm{n}}\right\|}{\left\|{ }_{\mathrm{s}} \mathbf{x}^{\mathrm{n}}\right\|}, \quad r=2, \ldots, R-2,
\end{gathered}
$$




$$
\frac{\left\|{ }_{\mathrm{s}}^{r} \mathbf{x}^{\mathrm{n}}\right\|}{\left\|{ }_{\mathrm{s}}^{R} \mathbf{x}^{\mathrm{n}}\right\|} \leq \frac{\left\|\mathbf{x}^{\mathrm{n}}\right\|}{\left\|{ }_{\mathrm{s}}^{R} \mathbf{x}^{\mathrm{n}}\right\|}, \quad r=R-1 .
$$

\section{3. ОЦЕНИВАНИЕ МАТРИЦ КУСОЧНО-НЕПРЕРЫВНОЙ МОДЕЛИ НЕЛИНЕЙНОГО ОБЪЕКТА}

Для оценивания матриц кусочнонепрерывной модели, соответствующей $R$ соседним установившимся режимам, для которых известны вектора нормированных установившихся значений переменных управления и переменных состояния

$$
{ }_{\mathrm{s}}^{r} \mathbf{u}^{\mathrm{n}},{ }_{\mathrm{s}}^{r} \mathbf{x}^{\mathrm{n}}, r=1,2, \ldots, R
$$

используются переходные процессы нелинейной модели объекта, то есть наборы значений нормированного вектора состояния

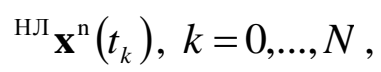

а также нормированного вектора управления

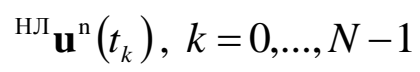

в дискретные моменты времени $t_{0}, t_{1}, t_{2}, \ldots, t_{k}, t_{k+1}, \ldots$, причем $t_{k+1}=t_{k}+\Delta t$, где $\Delta t$ это шаг дискретности.

Переходные процессы для переменных управления в разомкнутой системе управления представляют собой несколько последовательных ступенчатых воздействий, включающих в себя линейные функции времени, наклоны которых примерно соответствует переходным процессам в замкнутой системе управления. Эти линейные функции соединяют значения, соответствующие нескольким соседним установившимся режимам.

Оценивание матриц кусочно-непрерывной модели может быть сведено к задаче нелинейного программирования вида
${ }^{r} \mathbf{A},{ }^{r} \mathbf{B}, r=1,2, \ldots, R:$

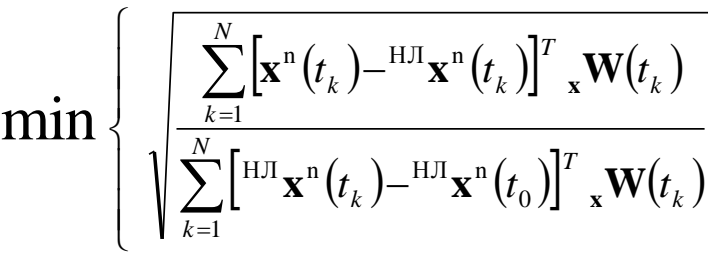

$$
\begin{aligned}
& {\left[{ }^{\text {нл }} \mathbf{x}^{\mathrm{n}}\left(t_{k}\right)-\mathbf{x}^{\mathrm{n}}\left(t_{k}\right)\right]} \\
& {\left[{ }^{\text {нл }} \mathbf{x}^{\mathrm{n}}\left(t_{k}\right)-{ }^{\text {нЛ }} \mathbf{x}^{\mathrm{n}}\left(t_{0}\right)\right]} \\
& \mathbf{x}^{\mathrm{n}}\left(t_{k+1}\right)=\mathbf{x}^{\mathrm{n}}\left(t_{k}\right)+ \\
& \Delta t \mathbf{A}\left[\mathbf{x}^{\mathrm{n}}\left(t_{k}\right)-{ }_{\mathrm{s}} \mathbf{x}^{\mathrm{n}}\right]+\Delta t \mathbf{B}\left[{ }^{\mathrm{HЛ}} \mathbf{u}^{\mathrm{n}}\left(t_{k}\right)-{ }_{\mathrm{s}} \mathbf{u}^{\mathrm{n}}\right], \\
& { }_{\mathrm{s}} \mathbf{u}^{\mathrm{n}}={ }_{\mathrm{s}}^{r} \mathbf{u}^{\mathrm{n}}+\theta\left({ }_{\mathrm{s}}^{r+1} \mathbf{u}^{\mathrm{n}}-{ }_{\mathrm{s}}^{r} \mathbf{u}^{\mathrm{n}}\right), \\
& { }_{\mathrm{s}} \mathbf{x}^{\mathrm{n}}={ }_{\mathrm{s}}^{r} \mathbf{x}^{\mathrm{n}}+\theta\left({ }_{\mathrm{s}}^{r+1} \mathbf{x}^{\mathrm{n}}-{ }_{\mathrm{s}}^{r} \mathbf{x}^{\mathrm{n}}\right), \\
& \mathbf{A}={ }^{r} \mathbf{A}+\theta\left({ }^{r+1} \mathbf{A}-{ }^{r} \mathbf{A}\right) \text {, } \\
& \mathbf{B}={ }^{r} \mathbf{B}+\theta\left({ }^{r+1} \mathbf{B}-{ }^{r} \mathbf{B}\right), \\
& \boldsymbol{\theta}=\frac{\left\|\mathbf{x}^{\mathrm{n}}\right\|-\left\|{ }_{\mathrm{s}}^{r} \mathbf{x}^{\mathrm{n}}\right\|}{\left\|{ }_{\mathrm{s}}^{r+1} \mathbf{x}^{\mathrm{n}}\right\|-\left\|{ }_{\mathrm{s}}^{r} \mathbf{x}^{\mathrm{n}}\right\|}, \\
& \frac{\left\|\mathbf{x}^{\mathrm{n}}\right\|}{\left\|{ }_{\mathrm{S}}^{R} \mathbf{x}^{\mathrm{n}}\right\|} \leq \frac{\left\|{ }_{\mathrm{s}}^{r+1} \mathbf{x}^{\mathrm{n}}\right\|}{\left\|{ }_{\mathrm{S}}^{R} \mathbf{x}^{\mathrm{n}}\right\|}, \quad r=1, \\
& \frac{\left\|{ }_{\mathrm{s}}^{r} \mathbf{x}^{\mathrm{n}}\right\|}{\left\|{ }_{\mathrm{s}}^{R} \mathbf{x}^{\mathrm{n}}\right\|} \leq \frac{\left\|\mathbf{x}^{\mathrm{n}}\right\|}{\left\|{ }_{\mathrm{s}}^{R} \mathbf{x}^{\mathrm{n}}\right\|} \leq \frac{\left\|{ }_{\mathrm{s}}^{r+1} \mathbf{x}^{\mathrm{n}}\right\|}{\left\|{ }_{\mathrm{s}}^{R} \mathbf{x}^{\mathrm{n}}\right\|}, \quad r=2, \ldots, R-2, \\
& \frac{\left\|{ }_{\mathrm{s}}^{r} \mathbf{x}^{\mathrm{n}}\right\|}{\|{ }_{\mathrm{s}}^{R} \mathbf{x}^{\mathrm{n} \|}} \leq \frac{\left\|\mathbf{x}^{\mathrm{n}}\right\|}{\left\|{ }_{\mathrm{s}}^{R} \mathbf{x}^{\mathrm{n}}\right\|}, \quad r=R-1, \\
& k=0,1, \ldots, N-1\} \text {. }
\end{aligned}
$$

Здесь $\quad \mathbf{\mathbf { W }}\left(t_{k}\right), \quad k=1, \ldots, N \quad$ положительно определенные диагональные весовые матрицы, а начальное значение вектора состояния кусочно-непрерывной модели

$$
\mathbf{x}^{\mathrm{n}}\left(t_{0}\right)={ }^{\mathrm{H} Л} \mathbf{x}^{\mathrm{n}}\left(t_{0}\right) .
$$

Задача нелинейного программирования решается методом случайного поиска. В качестве начального приближения используются матрицы кусочно-линейной 
модели [1]. Случайный поиск осуществляется в диапазоне \pm 0.1 от начального значения каждого из элементов матриц ${ }^{r} \mathbf{A},{ }^{r} \mathbf{B}, r=1,2, \ldots, R$.

Ошибки кусочно-непрерывной модели рассчитываются по каждой из переменных состояния

$$
{ }_{i} \Delta_{\infty}=\max _{k=1, \ldots, N}\left|x_{i}^{\mathrm{n}}\left(t_{k}\right)-{ }^{\mathrm{H} Л} x_{i}^{\mathrm{n}}\left(t_{k}\right)\right|, i=1, \ldots, n .
$$

\section{4. ЧИСЛЕННЫЙ ПРИМЕР ОЦЕНИВАНИЯ МАТРИЦ КУСОЧНО- НЕПРЕРЫВНОЙ МОДЕЛИ}

Разработанный метод применяется для определения матриц кусочно-непрерывной модели по переходным процессам нелинейной модели двухвального двухконтурного турбореактивного авиационного ГТД в разомкнутой САУ. Переходные процессы нелинейной модели начинаются на установившемся режиме, соответствующем максимальному режиму работы при значении угла отклонения рычага управления двигателем $\alpha_{\text {руд }}=68^{\circ}$, а затем соответствуют переходным процессам для переменных управления в разомкнутой системе, которые представляют собой несколько последовательных ступенчатых воздействий. Каждое из этих воздействий соответствует изменению значения угла отклонения рычага управления двигателем $\alpha_{\text {руд }}=60^{\circ}, \alpha_{\text {руд }}=50^{\circ}, \alpha_{\text {руд }}=40^{\circ}, \alpha_{\text {руд }}=30^{\circ}$, $\alpha_{\text {руд }}=25^{\circ}$.

В рассматриваемом примере высота и скорость полета равны нулю. Четыре переменные управления $(m=4)$ соответствуют расходу топлива в основной камере сгорания $G_{\text {то }}$, площади критического сечения реактивного сопла $F_{\text {кр }}$, углу поворота направляющих аппаратов вентилятора $\varphi_{\text {нав }}$ и углу поворота направляющих аппаратов компрессора $\varphi_{\text {нак }}$.
Пять переменных состояния объекта управления $(n=5)$ соответствуют частоте вращения вентилятора двигателя $n_{\text {в }}$, частоте вращения компрессора двигателя $n_{\text {к }}$, давлению торможения за компрессором $P_{\mathrm{\kappa}}^{*}$, давлению торможения за турбиной $P_{\text {т }}^{*}$ и температуре торможения за турбиной $T_{\mathrm{T}}^{*}$.

Вектора нормированных установившихся значений управления и состояния для перечисленных выше режимов

$$
\begin{aligned}
& { }_{\mathrm{s}}^{6} \mathbf{u}^{\mathrm{n}}=[1,0.4515,0,0]^{T}, \\
& { }_{\mathrm{s}}^{6} \mathbf{x}^{\mathrm{n}}=[1,1,1,1,1]^{T}, \\
& { }_{\mathrm{s}}^{5} \mathbf{u}^{\mathrm{n}}=[0.9442,0.4441,-0.0893,0]^{T}, \\
& { }_{\mathrm{s}}^{5} \mathbf{x}^{\mathrm{n}}=[0.9741,0.9834,0.9656,0.9783,0.9790]^{T}, \\
& { }_{\mathrm{s}}^{4} \mathbf{u}^{\mathrm{n}}=[0.7713,0.4441,-0.3741,0]^{T}, \\
& { }_{\mathrm{s}}^{4} \mathbf{x}^{\mathrm{n}}=[0.9176,0.9414,0.8355,0.8618,0.9225]^{T}, \\
& { }_{\mathrm{s}}^{3} \mathbf{u}^{\mathrm{n}}=[0.5724,0.4441,-0.7402,-0.1072]^{T}, \\
& { }_{\mathrm{s}}^{3} \mathbf{x}^{\mathrm{n}}=[0.8449,0.8991,0.6724,0.7125,0.8507]^{T}, \\
& { }_{\mathrm{s}}^{2} \mathbf{u}^{\mathrm{n}}=[0.2880,0.4441,-1,-0.2264]^{T}, \\
& { }_{\mathrm{s}}^{2} \mathbf{x}^{\mathrm{n}}=[0.7231,0.8399,0.4505,0.4787,0.6900]^{T}, \\
& { }_{\mathrm{s}}^{1} \mathbf{u}^{\mathrm{n}}=[0.1864,0.4441,-1,-0.4019]^{T}, \\
& { }_{\mathrm{s}}^{1} \mathbf{x}^{\mathrm{n}}=[0.6202,0.7929,0.3348,0.3788,0.6114]^{T} .
\end{aligned}
$$

Матрицы кусочно-непрерывной модели, соответствующие рассматриваемым соседним установившимся режимам, оцениваются с помощью (10) методом случайного поиска

$$
{ }^{6} \mathbf{A}=\left[\begin{array}{ccc}
-1.8208 & 1.1770 & \\
0.0697 & -1.6507 & \\
9.7385 & 11.5191 & \\
9.6711 & 3.5115 & \\
2.9867 & -6.8163 & \\
0 & 0 & 0 \\
0 & 0 & 0 \\
-20.1171 & -13.1868 & 0 \\
-5.5917 & -19.3568 & 0 \\
-3.4159 & -3.1171 & -30.0422
\end{array}\right],
$$


${ }^{6} \mathbf{B}=$
$\left[\begin{array}{cccc}0.6093 & 0.8201 & -0.1387 & 0.1788 \\ 0.4454 & 0.5736 & -0.0387 & -0.2204 \\ 10.8333 & -40.9063 & 2.6207 & 1.7204 \\ 7.1854 & -39.0036 & 2.1503 & 0.5060 \\ 16.0831 & 3.7726 & -0.8053 & -1.2318\end{array}\right]$,

$$
{ }^{5} \mathbf{A}=\left[\begin{array}{ccc}
-2.0989 & 1.0320 & \\
0.1873 & -1.8560 & \\
9.7914 & 11.5725 & \\
9.7835 & 3.6756 & \\
2.8621 & -6.9600 & \\
0 & 0 & 0 \\
0 & 0 & 0 \\
-20.0301 & -13.1503 & 0 \\
-5.5720 & -19.2893 & 0 \\
-3.4286 & -3.2235 & -29.9508
\end{array}\right],
$$

${ }^{5} \mathbf{B}=$

$\left[\begin{array}{cccc}0.5647 & 2.2715 & -0.4239 & 0.2514 \\ 0.6004 & 0.5737 & 0.1194 & -0.2008 \\ 14.2718 & -47.2595 & 2.2365 & -1.5488 \\ 9.2314 & -44.3070 & 2.4864 & -2.1365 \\ 17.9006 & 1.8247 & -0.4124 & -1.6880\end{array}\right]$,

$$
{ }^{4} \mathbf{A}=\left[\begin{array}{ccc}
-1.6270 & 1.2922 & \\
0.0649 & -2.0180 & \\
11.2399 & 12.6572 & \\
10.4333 & 4.6735 & \\
2.8845 & -6.9473 & \\
0 & 0 & 0 \\
0 & 0 & 0 \\
-19.7169 & -11.5759 & 0 \\
-5.0344 & -18.7211 & 0 \\
-3.4493 & -3.2843 & -29.9813
\end{array}\right],
$$

$$
{ }^{3} \mathbf{A}=\left[\begin{array}{ccc}
-2.3519 & 1.7300 & \\
0.1079 & -2.8400 & \\
13.2768 & 14.6302 & \\
9.7373 & 4.2626 & \\
2.8276 & -3.5453 & \\
0 & 0 & 0 \\
0 & 0 & 0 \\
-18.6061 & -10.3892 & 0 \\
-4.2626 & -15.6408 & 0 \\
-3.5453 & -3.2605 & -30.0025
\end{array}\right],
$$

${ }^{3} \mathbf{B}=$

$\left[\begin{array}{cccc}0.5980 & 1.8505 & -0.1297 & 0.1247 \\ 0.7252 & -0.1001 & -0.1947 & -0.3271 \\ 13.2790 & -20.5240 & 0.0531 & 1.8069 \\ 9.1333 & -19.3408 & -0.2446 & 1.4193 \\ 25.5911 & -9.0120 & -1.0117 & -3.2289\end{array}\right]$,

${ }^{2} \mathbf{A}=\mid \begin{array}{cc}-1.7279 & 1.9401 \\ 0.5693 & -2.5114 \\ 12.8263 & 14.9954 \\ 11.4961 & 6.4707 \\ 2.8544 & -6.8024\end{array}$

$$
\left.\begin{array}{ccc}
0 & 0 & 0 \\
0 & 0 & 0 \\
-18.7520 & -11.9884 & 0 \\
-4.5819 & -17.7373 & 0 \\
-3.4658 & -3.2515 & -29.9919
\end{array}\right]
$$

${ }^{2} \mathbf{B}=$

$\left[\begin{array}{cccc}0.9329 & 0.4549 & 0.1908 & 0.0990 \\ 0.5745 & -0.0184 & -0.1399 & -0.1354 \\ 11.4558 & -10.6185 & -0.1116 & 2.1666 \\ 6.7190 & -12.0750 & -0.2529 & 1.0489 \\ 32.4494 & -3.5233 & 2.0774 & -2.2863\end{array}\right]$

$$
{ }^{1} \mathbf{A}=\left[\begin{array}{ccc}
-1.9944 & 0.9354 & \\
1.2707 & -2.6249 & \\
12.5384 & 14.7670 & \\
11.4520 & 6.6307 & \\
2.0775 & -7.2209 & \\
0 & 0 & 0 \\
0 & 0 & 0 \\
-18.6298 & -11.9759 & 0 \\
-4.5503 & -17.6851 & \\
-4.9486 & -3.4682 & -29.4721
\end{array}\right],
$$




${ }^{1} \mathbf{B}=$
$\left[\begin{array}{cccc}0.7743 & 1.1148 & -0.2212 & -0.0727 \\ 1.4304 & -0.5693 & 0.2746 & -0.0064 \\ 14.3639 & -10.6913 & -2.1411 & 1.2968 \\ 8.7236 & -12.4513 & -0.8109 & 0.8420 \\ 38.9908 & -2.4710 & -0.1666 & 1.5486\end{array}\right]$.

При оценивании $k=1, \ldots, 2001, \Delta t=0.025$, а весовые матрицы единичные.

На рис. 1 показаны переходные процессы в разомкнутой САУ нормированной переменной состояния $x_{5}^{\mathrm{n}}$ нелинейной модели и кусочно-непрерывной модели, которая описывает нелинейный объект в окрестностях пяти соседних установившихся режимов.

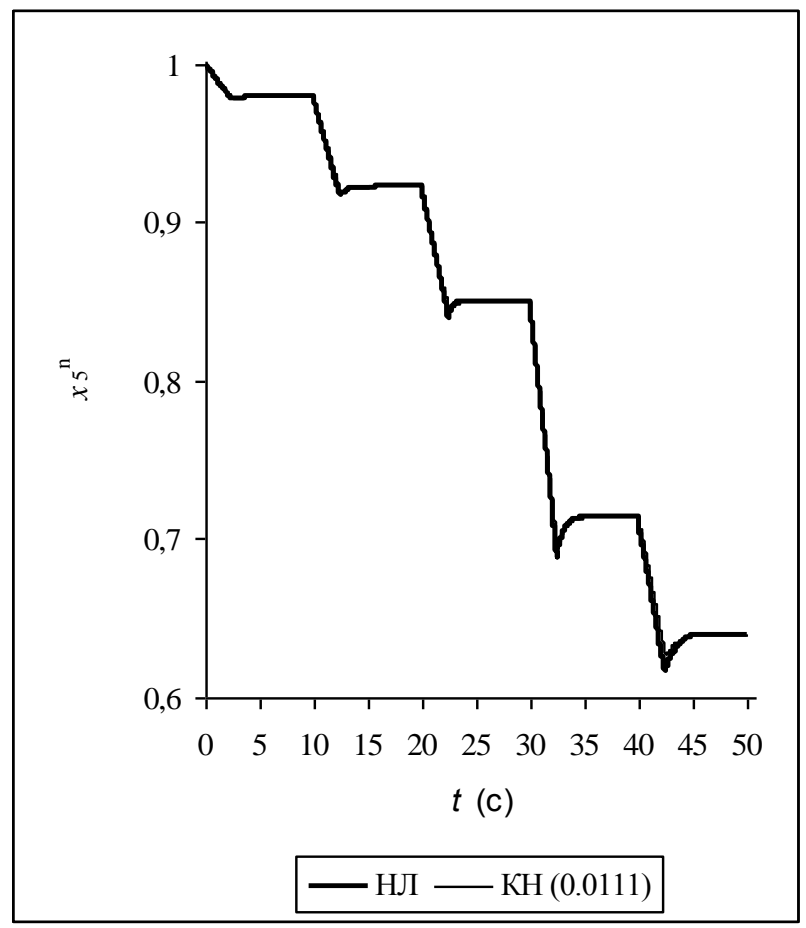

Рисунок 1. Переходные процессы в разомкнутой САУ нормированной переменной состояния $x_{5}^{n}$ нелинейной (НЛ) модели и кусочно-непрерывной (КН) модели, которая описывает нелинейный объект в окрестностях пяти соседних установивиихся режимов.
Ошибки нормированных переменных состояния $x_{1}^{\mathrm{n}}, x_{2}^{\mathrm{n}}, x_{3}^{\mathrm{n}}, x_{4}^{\mathrm{n}}$ и $x_{5}^{\mathrm{n}}$ кусочнонепрерывной модели в разомкнутой САУ $0.0108,0.0026,0.0057,0.0111$.

На рис. 2, 3, 4, 5, 6 показаны переходные процессы в замкнутой САУ нормированных переменных состояния $x_{1}^{\mathrm{n}}, x_{2}^{\mathrm{n}}, x_{3}^{\mathrm{n}}, x_{4}^{\mathrm{n}}$ и $x_{5}^{\mathrm{n}}$ нелинейной и кусочно-непрерывной моделей при изменению значения угла отклонения рычага управления двигателем от $\alpha_{\text {руд }}=68^{\circ}$ до $\alpha_{\text {руд }}=25^{\circ}$, а затем от $\alpha_{\text {руд }}=25^{\circ}$ до $\alpha_{\text {руд }}=68^{\circ}$.

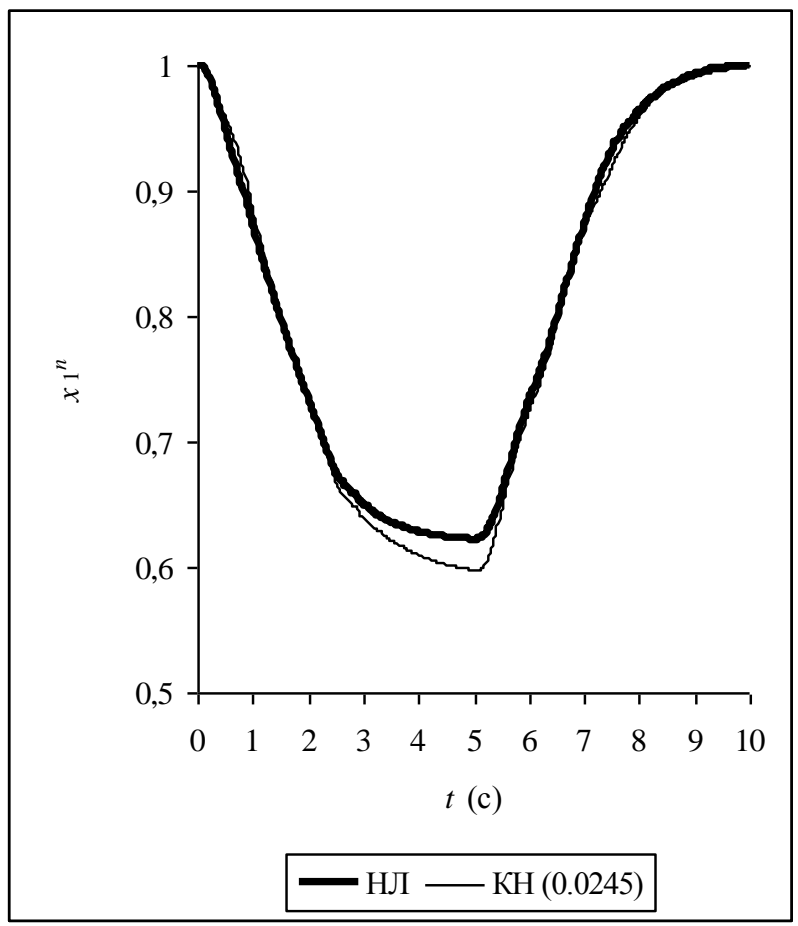

Рисунок 2. Переходные проиессы в замкнутой САУ нормированной переменной состояния $x_{1}^{n}$ нелинейной (НЛ) модели и кусочно-непрерывной (КН) модели в окрестностях пяти соседних установившихся режсиов. 


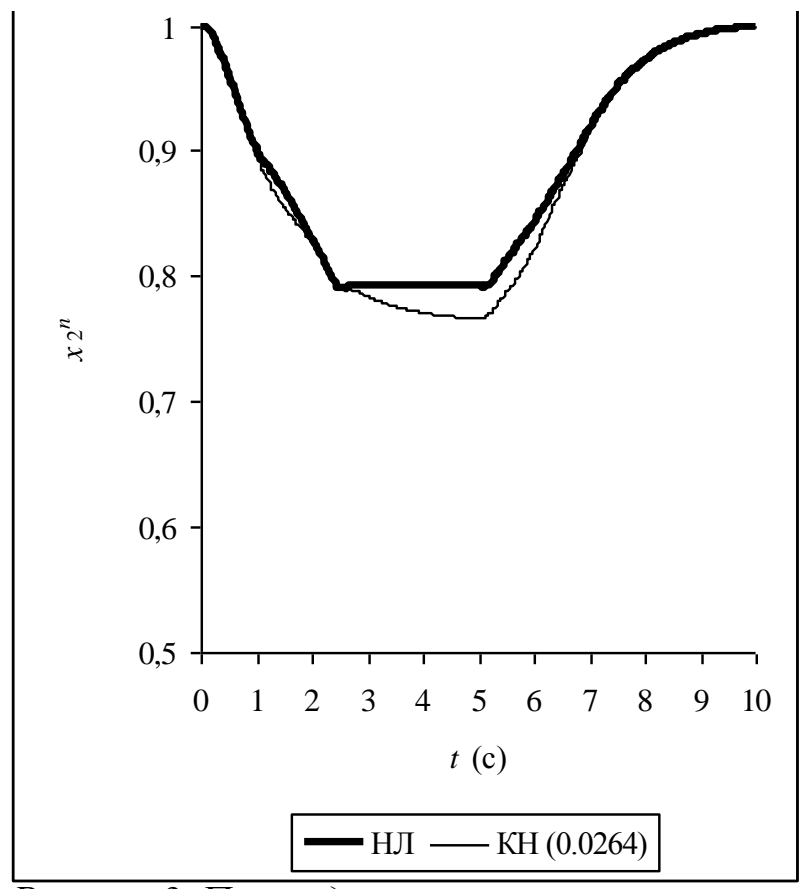

Рисунок 3. Переходные проиессы в замкнутой САУ нормированной переменной состояния

$x_{2}^{n}$ нелинейной (НЛ) модели и кусочнонепрерывной $(\mathrm{KH})$ модели в окрестностях пяти соседних установившихся режимов.

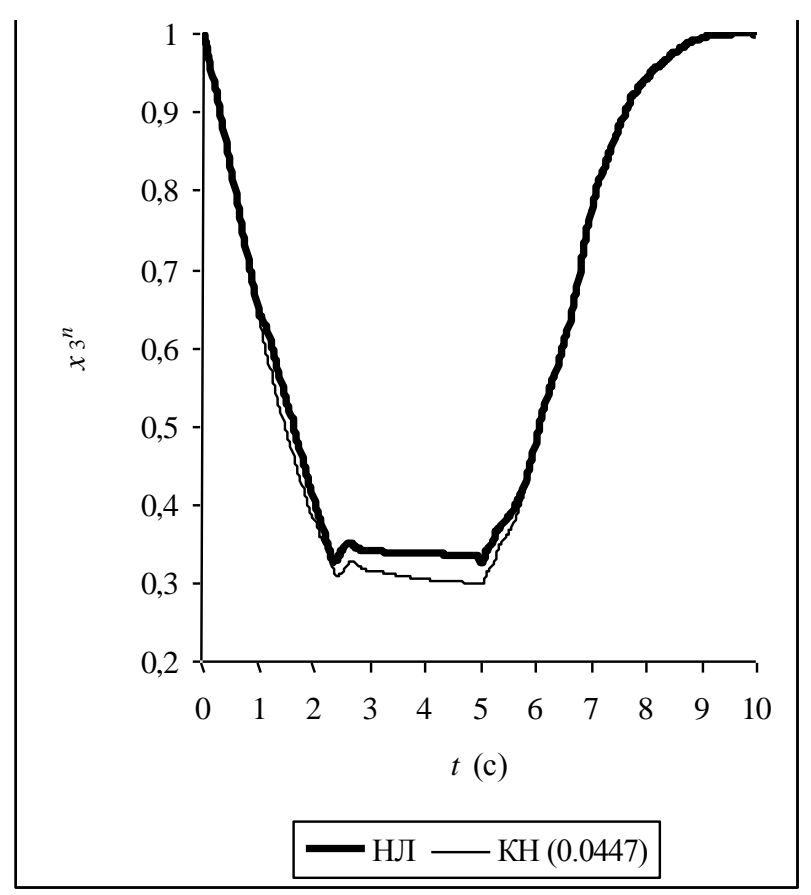

Рисунок 4. Переходные процессьи в замкнутой САУ нормированной переменной состояния $x_{3}^{n}$ нелинейной (НЛ) модели и кусочно-непрерывной (КН) модели в окрестностях пяти соседних установившихся режимов.

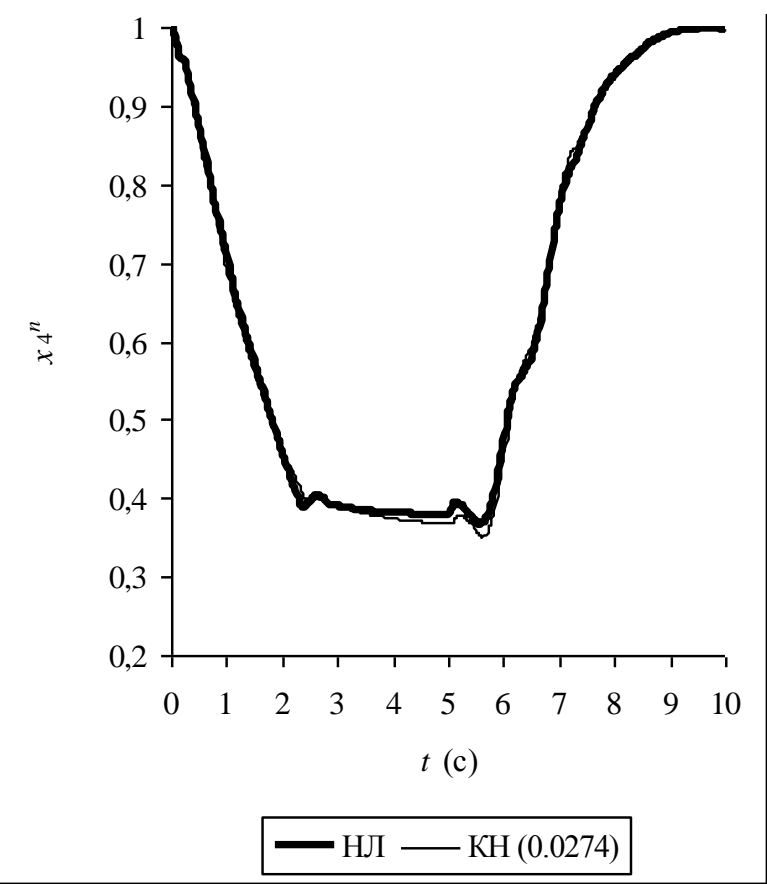

Рисунок 5. Переходные процессы в замкнутой САУ нормированной переменной состояния

$x_{4}^{n}$ нелинейной (НЛ) модели и кусочнонепрерывной (KH) модели в окрестностях пяти соседних установившихся режимов.

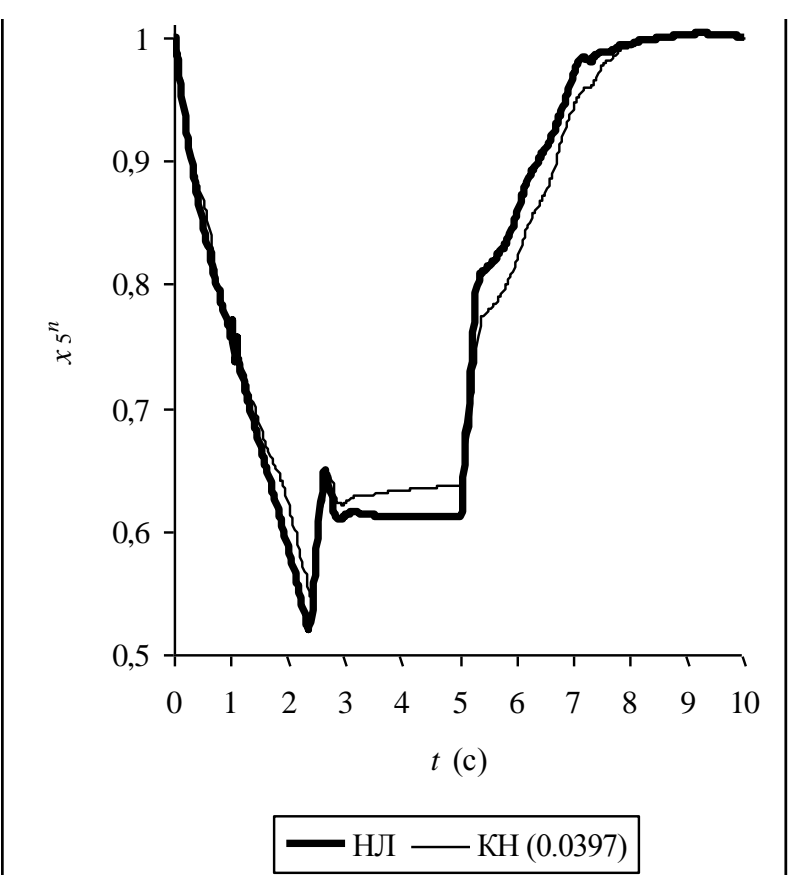

Рисунок 6. Переходные проиессы в замкнутой САУ нормированной переменной состояния $x_{5}^{n}$ нелинейной (НЛ) модели и кусочнонепрерывной (КН) модели в окрестностях пяти соседних установившихся режимов. 


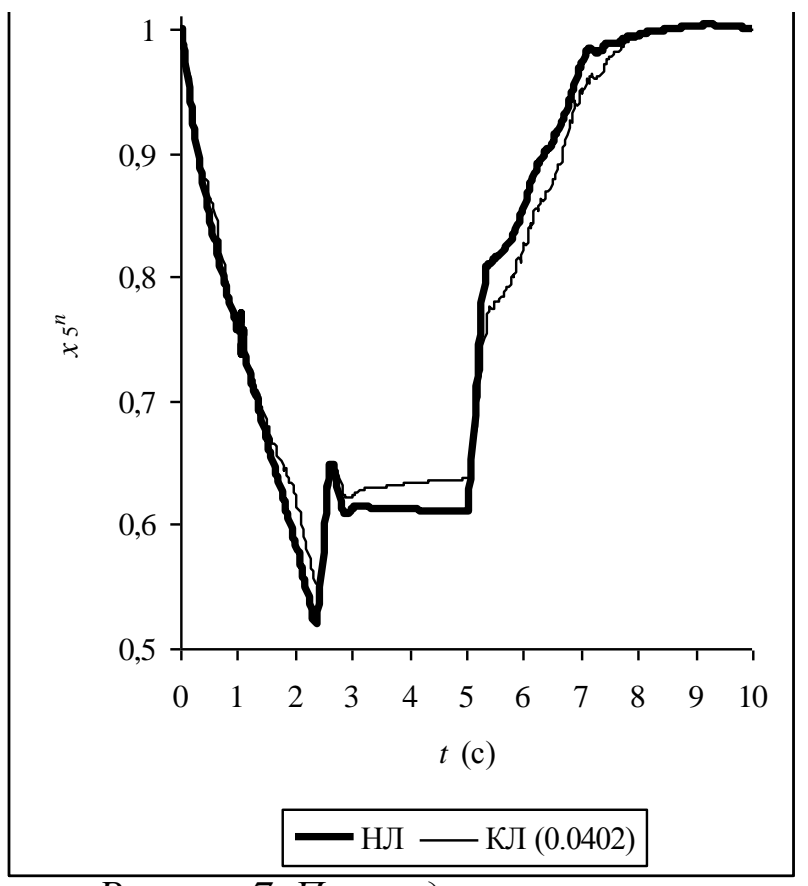

Рисунок 7. Переходные проиессы в замкнутой САУ нормированной переменной состояния $x_{5}^{n}$ нелинейной (НЛ) модели и кусочно-линейной (КЛ) модели, полученной из кусочно-непрерывной модели через каждые 0.1 с, в окрестностях пяти соседних установившихся режимов.

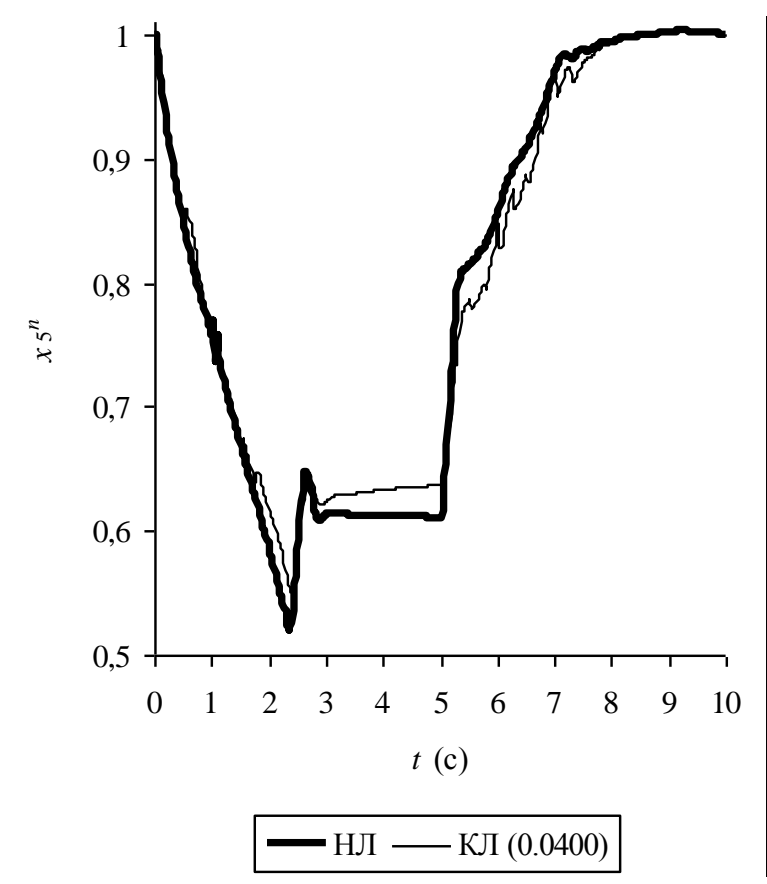

Рисунок 8. Переходные процессы в замкнутой САУ нормированной переменной состояния $x_{5}^{n}$ нелинейной (НЛ) модели и кусочно-линейной (КЛ) модели, полученной из кусочно-непрерывной модели через каждые 0.25 с, в окрестностях пяти соседних установившихся режимов.

На рис. 7, 8 показаны переходные процессы в замкнутой САУ нормированной переменной состояния $x_{5}^{\mathrm{n}}$ нелинейной модели и кусочно-линейной модели, полученной из кусочно-непрерывной модели.

Для получения кусочно-линейных моделей из кусочно-непрерывной модели через каждые 0.1 с и 0.25 с фиксируются вектора нормированных установившихся значений переменных управления $\mathbf{s}^{\mathrm{n}}$ и переменных состояния ${ }_{s} \mathbf{x}^{\mathrm{n}}$, а также матрицы $\mathbf{A}$ и $\mathbf{B}$ кусочно-непрерывной модели. При увеличении этого интервала ошибки нормированной переменной состояния $x_{5}^{\mathrm{n}}$ кусочно-линейной модели растут и становятся неприемлемыми. Ошибки других нормированных переменных состояния кусочно-линейной модели растут гораздо медленнее.

Ошибки кусочно-непрерывной и кусочнолинейной моделей по каждой из переменных состояния рассчитываются с помощью (11).

\section{5. ЗАКЛЮЧЕНИЕ}

Разработана методика оценивания матричных параметров кусочнонепрерывной модели нелинейного объекта управления.

В качестве исходных данных для оценивания матриц кусочно-непрерывной модели с помощью алгоритма случайного поиска, используются переходные процессы переменных управления и переменных состояния нелинейной модели объекта.

Результаты данной работы могут быть применены при разработке алгоритмов управления нелинейными объектами, которые приближенно описываются с помощью кусочно-непрерывных и кусочно- 
линейных моделей, а также алгоритмов обнаружения отказов датчиков и исполнительных устройств в их САУ.

\section{СПИСОК ЛИТЕРАТУРЫ}

1. Лейбов Р.Л. Прикладные методы теории управления. - М.: АСВ, 2014. $192 \mathrm{c}$.

2. Лейбов Р.Л. Методика построения кусочно-линейной модели нелинейного объекта. // International Journal for Computational Civil and Structural Engineering, 2013, Volume 9, Issue 4, pp. 186-198.

3. Лейбов Р.Л. Использование билинейной модели для улучшения свойств кусочно-линейной модели нелинейного объекта управления. // International Journal for Computational Civil and Structural Engineering, 2015, Volume 11, Issue 2, pp. 107-130.

\section{REFERENCES}

1. Leibov R.L. Prikladnye Metody Teorii Upravlenija [Applied Methods of Control Theory]. Moscow, ASV Publishing House, 2014, 192 pages.

2. Leibov R.L. Metodika Postroenija Kusochno-Linejnoj Modeli Nelinejnogo Objekta [Method of Construction of Piecewise Linear Model of Nonlinear Object]. // International Journal for Computational Civil and Structural Engineering, 2013, Volume 9, Issue 4, pp. 186-198.

3. Lejbov R.L. Ispol'zovanie Bilinejnoj Modeli dlja Uluchshenija Svojstv Kusochno-Linejnoj Modeli Nelinejnogo Objekta Upravlenija [Use of a Bilinear Model to Improve the Properties of a Piecewise Linear Model of a Nonlinear Control Object]. // International Journal for Computational Civil and Structural
Engineering, 2015, Volume 11, Issue 2, pp. 107-130.

Лейбов Роман Львович, кафедра прикладной математики, Национальный исследовательский Московский государственный строительный университет; 129337, Россия, г. Москва, Ярославское шоссе, дом 26; кафедра систем автоматического управления летательных аппаратов, Московский авиационный институт (национальный исследовательский университет); 125993, Россия, г. Москва, Волоколамское шоссе, дом 4, тел./факс +7(499)183-59-94;

e-mail: r_leibov@mtu-net.ru.

Roman L. Leibov, Department of Applied Mathematics, National Research Moscow State University of Civil Engineering; 26, Yaroslavskoe Highway, 129337, Moscow, RUSSIA; Department of Control Systems, Moscow Aviation Institute (National Research University), 4, Volokolamskoe Highway, 125993, Moscow, RUSSIA, tel./fax +7(499)183-59-94;

e-mail: r_leibov@mtu-net.ru. 